\title{
Curva de aprendizado e flutuações a curto e longo prazos com perimetria de freqüência dupla (FDT)
}

\author{
Learning effect and long-and short-term fluctuations in frequency doubling perimetry
}

\author{
Rui Barroso Schimiti ${ }^{1}$ \\ Vital Paulino Costa ${ }^{2}$ \\ Telma Gondim Freitas ${ }^{3}$ \\ Leopoldo Magacho4 \\ Enyr Saran Arcieri ${ }^{4}$ \\ Newton Kara-José
}

\section{R E S U M O}

Objetivo: Avaliar as flutuações que ocorrem a curto e a longo prazos e a curva de aprendizado que ocorre em indivíduos normais submetidos à perimetria de freqüência dupla. Métodos: Vinte indivíduos normais foram submetidos à perimetria de freqüência dupla, durante quatro sessões, com intervalos semanais. Na última sessão, realizaram-se três exames seguidos. As médias de sensibilidade e as flutuações a curto e longo prazos foram calculadas. Foi também avaliada a curva de aprendizado, pela comparação dos valores de MD ("mean deviation") nas quatro sessões. Resultado: $\mathrm{Na}$ avaliação a curto prazo, a média total de sensibilidade foi 31,91 $\pm 1,20 \mathrm{~dB}$ e as médias de MD e PSD ("pattern standard deviation") foram 0,84 1,85 e 3,73 $\pm 1,55 \mathrm{~dB}$, respectivamente. A média total das flutuações a curto prazo foi $1,72 \pm 0,38 \mathrm{~dB}$. Na avaliação a longo prazo, a média total de sensibilidade foi $31,75 \pm 1,11 \mathrm{~dB}$ e as médias de MD e PSD foram 0,68 $\pm 1,90 \mathrm{~dB}$ e 3,67 \pm $0,10 \mathrm{~dB}$, respectivamente. A média total das flutuações a longo prazo foi $2,16 \pm 0,26 \mathrm{~dB}$. Os MDs médios da primeira, segunda, terceira e quarta sessões foram $0,11 \pm 2,14 \mathrm{~dB}, 0,47 \pm 1,64 \mathrm{~dB}, 1,16 \pm 1,62 \mathrm{~dB}$ e $0,98 \pm 1,92 \mathrm{~dB}$, respectivamente. Observou-se aumento significativo do MD na terceira e quarta sessões em relação à primeira sessão $(\mathrm{p}<0,05)$. Conclusão: As sensibilidades detectadas pela perimetria de freqüência dupla apresentam flutuação a curto e longo prazo. Observou-se também claro efeito do aprendizado que deve ser considerado quando se realiza esse tipo de exame pela primeira vez.

Descritores:Perimetria/métodos;Campos visuais/fisiologia;Aprendizado

\footnotetext{
${ }^{1}$ Médico Assistente do Setor de Glaucoma do Hospital das Clínicas da Universidade Estadual de Campinas (UNICAMP) e pós-graduando (nível Doutorado) da UNICAMP.

${ }^{2}$ Professor Livre Docente pela Faculdade de Medicina da Universidade de São Paulo (USP), Chefe do Setor de Glaucoma do Hospital da Clínicas da UNICAMP.

${ }^{3}$ Médica Estagiária do Setor de Glaucoma da UNICAMP.

${ }^{4}$ Médico pós-graduando da UNICAMP.

${ }^{5}$ Professor Titular de Oftalmologia, Faculdade de Ciências Médicas da Universidade Estadual de Campinas UNICAMP e Faculdade de Medicina da Universidade de São Paulo - USP.

Endereço para Correspondência: Rua Senador Souza Naves, 897 - Londrina - (PR) CEP 86010-170

E-mail: ruioft@ sercomtel.com.br

Recebido para publicação em 27.02.2002 Aceito para publicação em 07.10.2002
}

I N T R O D U Ç ̃̃ O

O conhecimento da variabilidade esperada para qualquer algorítmo ou metodologia introduzidos na prática perimétrica é fundamental para proporcionar uma interpretação precisa das anormalidades e eventuais progressões que ocorrem em avaliações do campo visual. Reforça essa necessidade o fato de áreas já comprometidas do campo visual apresentarem flutuações, a longo e curto prazos, de grandes amplitudes quando a perimetria acromática é empregada ${ }^{(1)}$.

Sabe-se também que indivíduos saudáveis que não têm experiência na realização de exames perimétricos podem apresentar pseudodefeitos de campo visual que podem ser confundidos com alterações proporcionadas por afecções oculares. Em geral, estes indivíduos mostram uma melhora do padrão do exame, à medida que se tornam mais familiarizados com o teste ${ }^{(2-3)}$. Sendo assim, a curva de aprendizado de um exame deve ser avaliada, para que se possa conhecer a partir de qual momento o paciente é capaz de nos fornecer resultados confiáveis. 
Na perimetria computadorizada, as variações de sensibilidade retiniana num determinado ponto durante um único exame são denominadas flutuação a curto prazo. Quando as variações ocorrem entre exames realizados em dias diferentes, são denominadas flutuação a longo prazo ${ }^{(4-6)}$. O conhecimento das variações fisiológicas de sensibilidade retiniana é fundamental para melhorar a precisão das nossas interpretações.

Investigações para diagnosticar o glaucoma numa fase mais precoce levaram à busca de alterações funcionais específicas de determinadas subpopulações de células retinianas, ainda não percebidas pelos exames convencionais. Isso levou ao desenvolvimento da perimetria azul-amarelo, da perimetria de movimento ("motion perimetry") e da perimetria de freqüência dupla ${ }^{(7-9)}$.

A perimetria de freqüência dupla ou "Frequency Doubling Perimetry" é baseada na percepção de uma seqüência de barras verticais apresentadas sob uma freqüência espacial baixa (menos que 1 ciclo / 1 grau) e sob uma alta freqüência temporal $(25 \mathrm{~Hz})$. A apresentação destes estímulos produz uma ilusão óptica de duplicação espacial do número de barras $^{(8)}$. Acredita-se que esta nova técnica mede a função de uma subpopulação das células ganglionares denominadas de células My, constituintes do sistema magnocelular ${ }^{(8-9)}$. O sistema magnocelular representa aproximadamente $10 \%$ das células ganglionares e as células My representam aproximadamente $25 \%$ do total de células desse sistema ${ }^{(10)}$. Ainda há controvérsia sobre o conceito de que as células My são lesadas mais precocemente no glaucoma. De qualquer forma, sugere-se que o comprometimento de células específicas (como as My), que estão distribuídas difusamente na superfície retiniana, poderia diminuir o fenômeno de redundância, impedindo que a disfunção de um grupo específico de células possa ser mascarada por células vizinhas $^{(10-13)}$. Essa modalidade perimétrica tem-se mostrado altamente promissora para utilização em triagens populacionais ${ }^{(14)}$.
O objetivo do presente estudo foi avaliar a flutuação da sensibilidade que ocorre a longo prazo (testes com intervalos semanais), a flutuação da sensibilidade que ocorre a curto prazo (testes com intervalos de 15 minutos com paciente já experiente) e a curva de aprendizado em indivíduos normais submetidos ao FDT.

MÉ TODOS

Foram recrutados 22 indivíduos normais entre os médicos residentes e funcionários voluntários no Departamento de Oftalmologia do Hospital das Clínicas da UNICAMP no período de novembro a dezembro de 2000. Os indivíduos foram submetidos à medida da acuidade visual, exame de biomicroscopia, oftalmoscopia direta e com lente de Volk +78 dioptrias esféricas (DE), tonometria de aplanação (Goldmann) e refração sob ciclopegia. Os critérios de inclusão para participar do estudo foram: acuidade visual $>$ ou $=$ a 0,8 , Po $<21 \mathrm{mmHg}$, relação escavação/disco $<$ ou $=$ a 0,5 , assimetria de escavação $<0,2$, erro de refração $<$ ou $= \pm 5,00$ dioptrias (equivalente esférico). Os critérios de exclusão foram: presença de hemorragias peri-papilares ou "notch", história de cirurgia intra-ocular ou ambliopia, história familiar positiva de glaucoma, antecedentes de doença ocular ou trauma e experiência prévia com FDT.

Com o intuito de avaliar a flutuação a longo prazo, os indivíduos foram submetidos à perimetria de freqüência dupla em quatro sessões realizadas semanalmente. Com a finalidade de avaliar a flutuação a curto prazo, procedeu-se à realização de três exames seguidos com intervalo de 15 minutos entre cada exame na última sessão, quando os indivíduos já estavam acostumados com o teste. Testou-se somente o olho direito de cada indivíduo com a correção para longe.

\begin{tabular}{|c|c|c|c|c|}
\hline $\mathbf{A}$ & $\begin{array}{l}31,73 \\
(5,44)\end{array}$ & $\begin{array}{l}32,90 \\
(4,47)\end{array}$ & $\begin{array}{l}32,02 \\
(3,59)\end{array}$ & $\begin{array}{l}31,75 \\
(3,48)\end{array}$ \\
\hline $\begin{array}{l}29,05 \\
(4,02)\end{array}$ & $\begin{array}{l}33,40 \\
(3,01)\end{array}$ & $\begin{array}{c}32,93 \\
(2,79)\end{array}$ & $\begin{array}{r}32,42 \\
(2,26) \\
\end{array}$ & $\begin{array}{l}31,73 \\
(3,26)\end{array}$ \\
\hline \multirow[t]{2}{*}{$\begin{array}{l}29,22 \\
(3,43)\end{array}$} & $\begin{array}{l}33,52 \\
(4,35)\end{array}$ & $\begin{array}{c}32,12 \\
(2,79)\end{array}$ & $\begin{array}{l}32,95 \\
(2,61)\end{array}$ & $\begin{array}{l}30,90 \\
(3,09)\end{array}$ \\
\hline & $\begin{array}{l}31,08 \\
(3,92)\end{array}$ & $\begin{array}{l}32,00 \\
(3,10)\end{array}$ & $\begin{array}{l}32,33 \\
(2,75)\end{array}$ & $\begin{array}{l}32,00 \\
(2,93\})\end{array}$ \\
\hline
\end{tabular}

Média total $( \pm \mathrm{DP})$ das sensibilidades $=31,91 \pm 1,20$

\begin{tabular}{|c|c|c|c|c|}
\hline B & $\begin{array}{c}2,32 \\
(2,84)\end{array}$ & $\begin{array}{c}2,35 \\
(2,55)\end{array}$ & $\begin{array}{c}1,73 \\
(1,33)\end{array}$ & $\begin{array}{c}1,29 \\
(1,06)\end{array}$ \\
\hline $\begin{array}{c}2,00 \\
(1,74)\end{array}$ & $\begin{array}{c}1,65 \\
(1,21)\end{array}$ & $\begin{array}{c}1,57 \\
(1,14)\end{array}$ & $\begin{array}{c}1,30 \\
(0,93)\end{array}$ & $\begin{array}{r}1,65 \\
(1,46)\end{array}$ \\
\hline \multirow[t]{2}{*}{$\begin{array}{c}1,84 \\
(1,34)\end{array}$} & $\begin{array}{c}2,30 \\
(2,35)\end{array}$ & $\begin{array}{c}1,48 \\
(1,24)\end{array}$ & $\begin{array}{c}1,43 \\
(1,25)\end{array}$ & $\begin{array}{c}1,67 \\
(1,19)\end{array}$ \\
\hline & $\begin{array}{c}2,13 \\
(1,84)\end{array}$ & $\begin{array}{c}2,00 \\
(1,09)\end{array}$ & $\begin{array}{c}1,27 \\
(1,06)\end{array}$ & $\begin{array}{c}1,25 \\
(0,96)\end{array}$ \\
\hline
\end{tabular}

Média total $( \pm$ DP) das flutuações $=1,72 \pm 0,38$ 
Os testes foram realizados com o "Frequency Doubling Technology Perimeter" (Welch Allyn, Skaneateles, New York, and Humphrey Systems, Dublin, California) ${ }^{(9)}$. Apesar de não haver interferência da luz ambiental (segundo orientações do fabricante) as luzes do ambiente permaneceram desligadas durante os testes. (No início da realização do teste, o aparelho cobre automaticamente o olho não testado). O indivíduo foi orientado a olhar no ponto de fixação e apertar o sinalizador manual a cada estímulo percebido. Utilizou-se o programa N30, que determina o limiar de sensibilidade de 19 áreas localizadas nos 30 graus centrais do campo visual, sendo uma área central de cinco graus de raio circundada por quatro áreas em cada um dos quatro quadrantes, doze áreas situadas entre os dez e vinte graus do ponto de fixação e duas áreas nasais, dispostas imediatamente acima e abaixo do meridiano horizontal (Figuras 2 e 3 ).

Os indivíduos foram analisados em relação ao sexo, cor, idade, acuidade visual e pressão intra-ocular (Po). Para avaliar a dispersão dos valores de sensibilidade, foram calculadas: 1) a média de sensibilidade de todos os pontos testados de cada indivíduo (sensibilidade média total); 2) a média dos limiares ponto a ponto de todos os exames (sensibilidade média focal). A flutuação a curto prazo ponto a ponto foi calculada como a média dos desvios-padrão das médias das sensibilidades encontradas em cada ponto nos três exames consecutivos da última sessão. A flutuação a longo prazo ponto a ponto foi calculada como a média dos desvios-padrão das médias das sensibilidades encontradas em cada ponto nas três primeiras sessões e no primeiro exame da quarta sessão. Também se calculou a média total das flutuações, tanto nas avaliações a longo como a curto prazo.

Para estudar o efeito aprendizado, o MD dos pacientes na primeira sessão foi comparado com o MD encontrado na segunda e terceira sessões e no primeiro exame da quarta sessão; da mesma forma, foram comparados o tempo de exame, o valor da sensibilidade central, PSD, a porcentagem de erros falsopositivos, falso-negativos e de perdas de fixação de cada exame, assim como o número de pontos classificados como presentes em menos do que $5 \%, 2 \%, 1 \%$ e $0,5 \%$ de população normal da mesma faixa etária (de acordo com a "database" do instrumento). As comparações foram realizadas com o emprego do teste de Wilcoxon para amostras pareadas, devido ao fato de a distribuição da maioria dos dados não ser normal e as amostras serem pareadas. Adotou-se o nível de significância de $5 \%$ em todos os testes estatísticos.

\section{RES U L T A DOS}

Dos vinte e dois indivíduos que preencheram os critérios de inclusão, vinte compareceram a todas as sessões necessárias para esse estudo. A média de idade foi de 28,1 $\pm 4,9$ anos (média \pm desvio-padrão) variando de 24 a 38 anos. $\mathrm{O}$ erro de refração (equivalente esférico) variou de -4,50 DE a +1,25 DE com valor médio de $-1,16 \pm 1,6 \mathrm{DE}$ e a acuidade visual encontrada foi 20/20 em todos os indivíduos. Dos indivíduos testados, $19(95 \%)$ eram brancos e $1(5 \%)$ negro, sendo $10(50 \%)$ mulheres e $10(50 \%)$ homens. A Po média dos vinte indivíduos analisados foi de $15,35 \pm 1,95 \mathrm{mmHg}$.

A tabela 1 mostra as médias de sensibilidade, MD, PSD, número de perdas de fixação, número de erros falso-positivos e número de erros falso-negativos dos quatro primeiros exames realizados (avaliação a longo prazo) e dos três exames realizados no mesmo dia (avaliação a curto prazo).

$\mathrm{Na}$ análise da flutuação a curto prazo, a média total de sensibilidade foi de $31,91 \pm 1,20 \mathrm{~dB}$ e as médias de MD e PSD foram de $0,84 \pm 1,85$ e de $3,73 \pm 1,55 \mathrm{~dB}$, respectivamente. A média total das flutuações encontradas foi de $1,72 \pm 0,38 \mathrm{~dB}$ e as médias de flutuações ponto a ponto variaram de 1,25 $\pm 0,96$ a 2,35 $\pm 2,55 \mathrm{~dB}$ (Figuras 1-A e 1-B).

\begin{tabular}{|c|c|c|c|c|}
\hline $\mathbf{A}$ & $\begin{array}{l}31,31 \\
(4,69)\end{array}$ & $\begin{array}{l}32,44 \\
(3,78)\end{array}$ & $\begin{array}{l}32,05 \\
(3,45)\end{array}$ & $\begin{array}{l}31,59 \\
(3,49)\end{array}$ \\
\hline $\begin{array}{l}29,01 \\
(3,80)\end{array}$ & $\begin{array}{c}33,43 \\
(3,40)\end{array}$ & $\begin{array}{l}33,09 \\
(2,96)\end{array}$ & $\begin{array}{l}32,35 \\
(2,94)\end{array}$ & $\begin{array}{l}31,31 \\
(3,46)\end{array}$ \\
\hline \multirow[t]{2}{*}{$\begin{array}{l}29,53 \\
(3,34)\end{array}$} & $\begin{array}{c}33,04 \\
(3,25)\end{array}$ & $\begin{array}{l}31,93 \\
(2,59)\end{array}$ & $\begin{array}{l}32,34 \\
(2,96)\end{array}$ & $\begin{array}{l}31,01 \\
(2,95)\end{array}$ \\
\hline & $\begin{array}{l}30,91 \\
(3,78)\end{array}$ & $\begin{array}{l}31,84 \\
(2,73)\end{array}$ & $\begin{array}{l}31,93 \\
(3,29)\end{array}$ & $\begin{array}{l}31,80 \\
(3,78)\end{array}$ \\
\hline
\end{tabular}

Média total $( \pm \mathrm{DP})$ das sensibilidades $=31,75 \pm 1,11 \mathrm{~dB}$

\begin{tabular}{|c|c|c|c|c|}
\hline B & $\begin{array}{c}2,66 \\
(2,10)\end{array}$ & $\begin{array}{c}2,43 \\
(1,64)\end{array}$ & $\begin{array}{c}2,19 \\
(0,89)\end{array}$ & $\begin{array}{c}2,21 \\
(1,14)\end{array}$ \\
\hline $\begin{array}{c}2,41 \\
(1,40)\end{array}$ & $\begin{array}{c}2,34 \\
(1,60)\end{array}$ & $\begin{array}{c}2,05 \\
(1,24)\end{array}$ & $\begin{array}{c}2,03 \\
(0,83)\end{array}$ & $\begin{array}{c}2,17 \\
(1,32)\end{array}$ \\
\hline \multirow[t]{2}{*}{$\begin{array}{c}2,52 \\
(0,94)\end{array}$} & $\begin{array}{c}2,11 \\
(1,00)\end{array}$ & $\begin{array}{c}1,86 \\
(0,87)\end{array}$ & $\begin{array}{c}1,92 \\
(1,14)\end{array}$ & $\begin{array}{c}1,80 \\
(1,09)\end{array}$ \\
\hline & $\begin{array}{c}2,17 \\
(1,25)\end{array}$ & $\begin{array}{c}1,79 \\
(0,82)\end{array}$ & $\begin{array}{c}2,11 \\
(1,46)\end{array}$ & $\begin{array}{c}2,41 \\
(1,29)\end{array}$ \\
\hline
\end{tabular}

Média total $( \pm \mathrm{DP})$ das flutuações $=2,16 \pm 0,26$ 


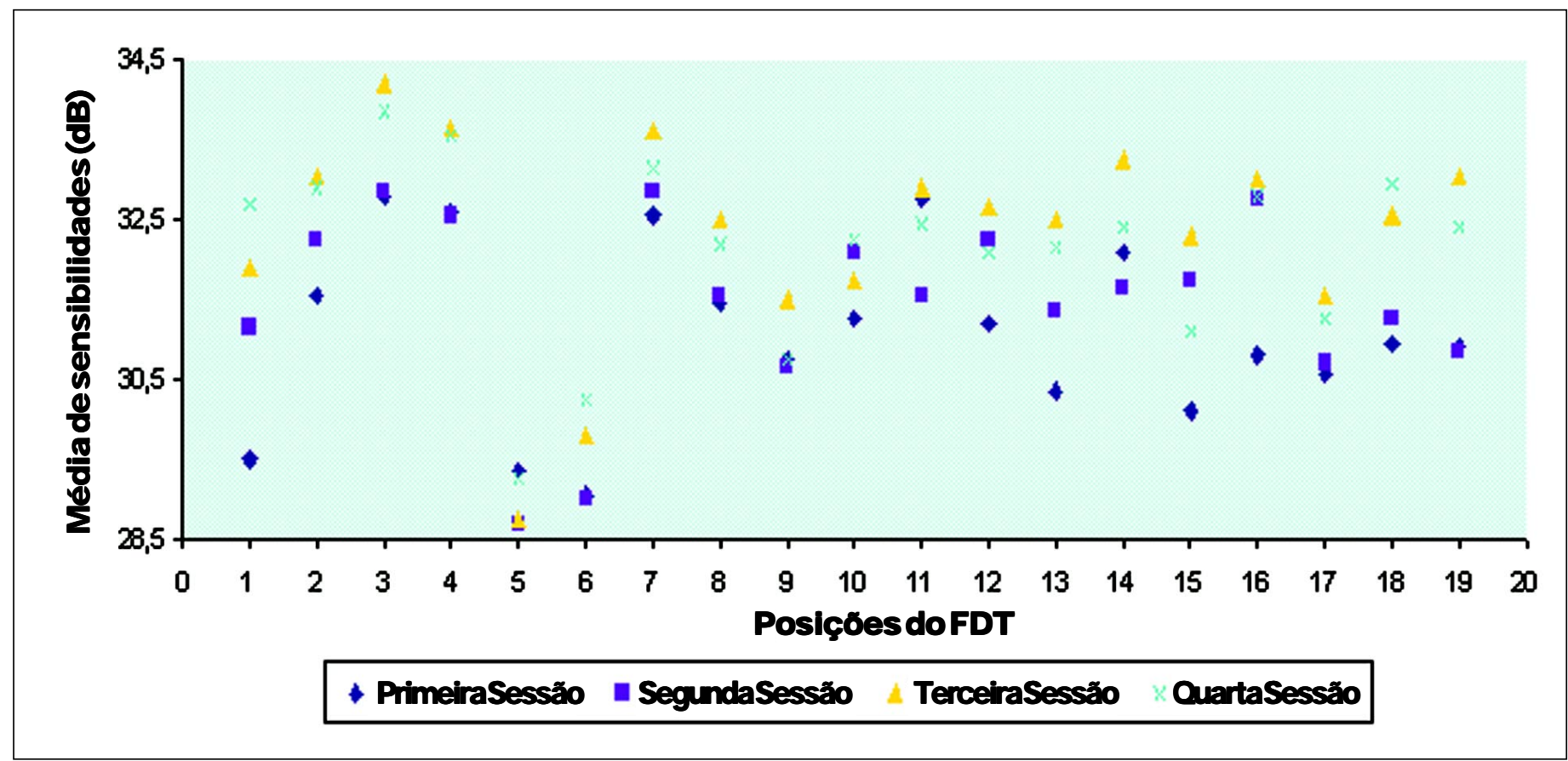

Figura 3-Médias desensibilidade paracada pontotestadonas 4sessões com FDT.As médias desensibilidadetendemaaumentarapósa primeira sessão, tomando-semais uniformes nas duas últimas sessöes. As posiçōes sãonumeradas na seguinte ordem: 1 a 4, quadrantenasal superior 5e6, pontos extremos nasais, superiore inferior, respectivamente;7 a 10, quadrante nasalinferior, 11 ponto central; 12 a 15, quadrantetemporal superior; 15 a 19, quadrante nasal inferior. Em cada um dos quadrantes, a ordem seqüencial éda esquerda para a direita e de cima para baixo

\begin{tabular}{|c|c|c|}
\hline & \multicolumn{2}{|c|}{ Média \pm DP } \\
\hline & Estudocurto prazo & Estudo longo prazo \\
\hline $\begin{array}{l}\text { Sensibilidade } \\
\text { média (dB) }\end{array}$ & $31,91 \pm 2,42$ & $31,75 \pm 2,43$ \\
\hline $\begin{array}{l}\text { MD (dB) } \\
\text { PSD (dB) }\end{array}$ & $\begin{array}{l}0,84 \pm 1,85 \\
3,73 \pm 1,55\end{array}$ & $\begin{array}{l}0,68 \pm 1,90 \\
3,67 \pm 0,10\end{array}$ \\
\hline PF (\%) & $6,50 \pm 13,30$ & $5,90 \pm 12,90$ \\
\hline FP (\%) & $2,60 \pm 5,50$ & $2,70 \pm 7,10$ \\
\hline FN (\%) & $0,00 \pm 0,00$ & $0,00 \pm 0,00$ \\
\hline
\end{tabular}

A tabela 2 revela as médias da duração do exame, da sensibilidade central, PSD, número de perdas de fixação, respostas falsopositivas, respostas falso-negativas e número de áreas alteradas com $\mathrm{p}<$ do que $5 \%, 2 \%, 1 \%$ e $0,5 \%$ dos quatro exames realizados semanalmente. Não houve diferença estatisticamente significativa quando se compararam estes parâmetros nas diversas sessões, com exceção do número de perdas de fixação do segundo exame que diferiu significativamente do primeiro exame $(\mathrm{p}=0,013)$.

$\mathrm{Na}$ análise de flutuação a longo prazo, a média total de sensibilidade foi de $31,75 \pm 1,11 \mathrm{~dB}$ e as médias de MD e PSD foram de $0,68 \pm 1,90 \mathrm{~dB}$ e de $3,67 \pm 0,10 \mathrm{~dB}$, respectivamente. A média total das flutuações encontradas foi de $2,16 \pm 0,26 \mathrm{~dB}$ e as médias de flutuações ponto a ponto variaram de 1,79 $\pm 1,47$ a 2,66 $\pm 2,10 \mathrm{~dB}$ (Figuras 2-A e 2-B).
Para que fosse analisada a validade da utilização da última sessão para melhor avaliar as flutuações a curto prazo, comparou-se a média das sensibilidades ponto a ponto em cada uma das quatro visitas. Os dados expressos na Figura 3 demonstram que a terceira e a quarta sessões apresentam em geral médias de sensibilidades ponto a ponto maiores.

Para avaliar o efeito aprendizado, compararam-se os valores médios de $\mathrm{MD}$ da primeira sessão $(0,11 \pm 2,14 \mathrm{~dB})$, da segunda sessão $(0,47 \pm 1,64 \mathrm{~dB})$, da terceira sessão $(1,16 \pm 1,62 \mathrm{~dB})$ e do primeiro exame da quarta $(0,98 \pm 1,92 \mathrm{~dB})$ sessão. Não houve diferença estatisticamente significativa entre o MD da primeira sessão e o MD da segunda sessão ( $p=0,2127)$ mas os MDs da terceira e da quarta sessões foram significativamente maiores que o MD da primeira sessão ( $p=0,0071$ e $p=0,0209$, respectivamente) (Figura 4).

\section{I S C U S S ÃO}

O FDT representa uma inovação na perimetria. Já se demonstrou que essa tecnologia é altamente promissora para triagens populacionais ${ }^{(8,10,14)}$, mas sua aplicação na clínica ainda tem sido objeto de estudo ${ }^{(13,15)}$. O conhecimento das flutuações que podem ocorrer em novos tipos de perimetria é de grande importância, tanto em condições normais quanto em situações em que já existe comprometimento do campo visual. Sabe-se que, na perimetria automatizada convencional, as flutuações podem ser influenciadas pela idade, opacidades de meios, inexperiência do indivíduo e doenças sistêmicas, mostrando-se 


\begin{tabular}{|c|c|c|c|c|}
\hline \multirow{3}{*}{$\begin{array}{l}\text { Duraçãomédia (s) } \\
\text { Sensibilidadecentral(dB) }\end{array}$} & \multirow{2}{*}{$\begin{array}{c}\text { Exame1 } \\
298,95 \pm 34,03\end{array}$} & \multirow{2}{*}{$\begin{array}{c}\text { Exame2 } \\
306,55 \pm 27,71\end{array}$} & Exame3 & Exame4 \\
\hline & & & $290,55 \pm 26,14$ & $288,90 \pm 25,72$ \\
\hline & $32,75 \pm 4,45$ & $31,55 \pm 3,07$ & $32,90 \pm 2,45$ & $32,45 \pm 2,31$ \\
\hline PSD (dB) & $3,92 \pm 1,11$ & $3,67 \pm 0,74$ & $3,44 \pm 0,70$ & $3,64 \pm 1,42$ \\
\hline PF (\%)* & $0,80 \pm 3,58$ & $6,50 \pm 11,14$ & $6,55 \pm 12,40$ & $9,85 \pm 18,88$ \\
\hline FP (\%) & $0,60 \pm 2,68$ & $4,90 \pm 8,42$ & $3,65 \pm 8,99$ & $1,85 \pm 6,07$ \\
\hline FN (\%) & $0,00 \pm 0,00$ & $0,00 \pm 0,00$ & $0,00 \pm 0,00$ & $0,00 \pm 0,00$ \\
\hline $\mathbf{P}<5 \%$ & $1,25 \pm 2,20$ & $0,55 \pm 1,61$ & $0,25 \pm 0,72$ & $0,40 \pm 1,19$ \\
\hline $\mathbf{P}<2 \%$ & $0,30 \pm 0,57$ & $0,30 \pm 1,13$ & $0,10 \pm 0,31$ & $0,15 \pm 0,49$ \\
\hline $\mathbf{P}<1 \%$ & $0,00 \pm 0,00$ & $0,05 \pm 0,22$ & $0,00 \pm 0,00$ & $0,05 \pm 0,22$ \\
\hline$P<0,5 \%$ & $0,00 \pm 0,00$ & $0,00 \pm 0,00$ & $0,00 \pm 0,00$ & $0,00 \pm 0,00$ \\
\hline \multicolumn{5}{|c|}{ 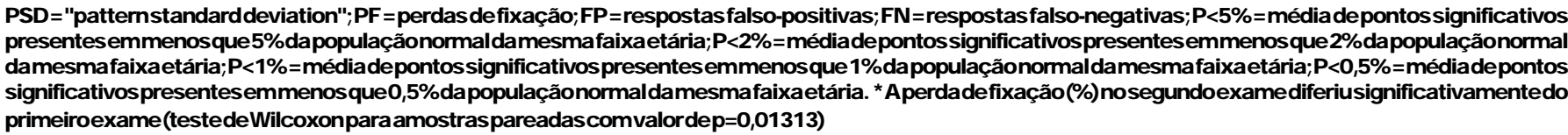 } \\
\hline
\end{tabular}

também aumentadas em áreas próximas a escotomas ${ }^{(16-17)}$. A distância em relação ao ponto de fixação central também pode influenciar a flutuação, tendo sido observado que na periferia ocorre uma maior variabilidade em relação às áreas mais centrais ${ }^{(17)}$. Flammer et al. ${ }^{(1)}$ descreveram uma flutuação a curto prazo de $1,6 \mathrm{~dB}$ e uma flutuação a longo prazo de $0,2 \mathrm{~dB}$, em indivíduos normais submetidos à perimetria computadorizada convencional. Utilizando o perímetro Humphrey, Katz e Sommer ${ }^{(18)}$ encontraram uma flutuação a longo prazo que variou de 1,8 a 5,5 dB dependendo da distância do ponto de fixação.

Estudos desenvolvidos por Iester et $\mathrm{al}^{(19)}$ encontraram valores similares de flutuações a longo e curto prazos no FDT, comparados com valores encontrados com perimetria convencional utilizando perímetro Octopus, embora os dois aparelhos envolvam estímulos distintos. Archibald et al. ${ }^{(20)}$ observaram médias de flutuação a curto prazo de 2,84 $\pm 1,13 \mathrm{~dB}$ e de flutuação a longo prazo de 2,17 $\pm 1,38 \mathrm{~dB}$ utilizando o FDT. Estudo que avaliou pacientes jovens normais submetidos ao FDT $^{(5)}$ encontrou flutuações a curto prazo que variaram de 1,4

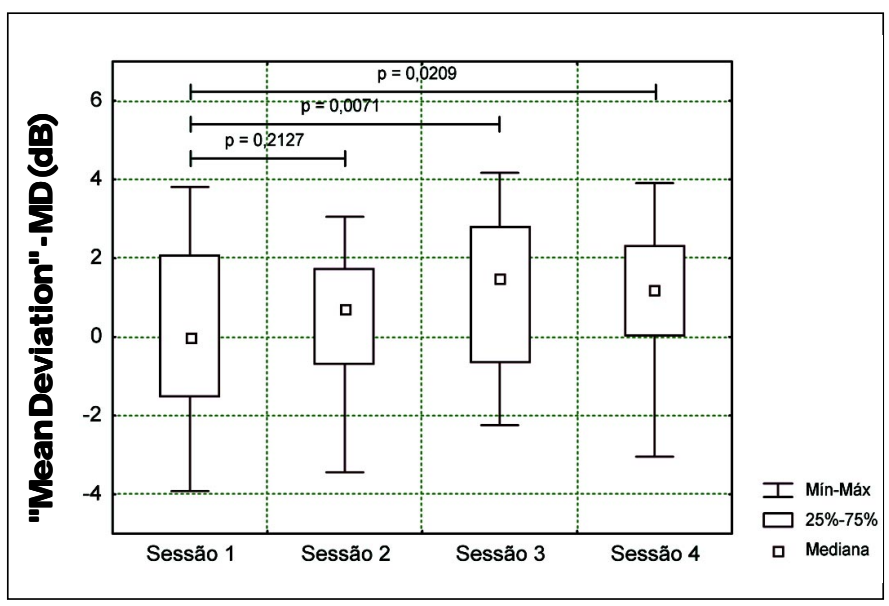

Figura 4-Efeitoaprendizadomostrandoumaumento progressivodo valordo "meandeviation" (MD) nas quatro sessốes a 3,4 dB, com média de 2,16 $\pm 0,5 \mathrm{~dB}$ e flutuações a longo prazo que variaram de 2,5 a 4,4 dB, com média de 3,23 $\pm 0,5 \mathrm{~dB}$. As médias de sensibilidade encontradas foram de $30,4 \pm 1,24 \mathrm{~dB}$ e de 32,4 $\pm 1,14 \mathrm{~dB}$ nas avaliações a curto e a longo prazos, respectivamente.

A média de flutuações a curto prazo encontrada no nosso estudo foi de $1,72 \pm 0,33 \mathrm{~dB}$ e a média de flutuações a longo prazo foi $2,16 \pm 0,33 \mathrm{~dB}$. As médias de flutuações encontradas por nós foram discretamente menores em relação ao estudo de Iester et al, tanto nas avaliações a curto como a longo prazos. Para isso não temos uma explicação clara, uma vez que ambos estudos avaliaram populações constituídas de indivíduos jovens pertencentes aos respectivos "staffs" dos Departamentos envolvidos. Na avaliação a curto prazo, é possível que a menor flutuação encontrada no presente estudo possa ser justificada pelo fato de os exames seqüenciados terem sido realizados somente na quarta sessão (portanto, com os indivíduos bem familiarizados com o teste), diferentemente do estudo de Iester que testou os indivíduos já da segunda sessão. Num estudo subseqüente avaliaremos estes valores em uma população idosa, mais compatível com a que utilizaria o exame para detecção do glaucoma. As médias de sensibilidade encontradas em nosso estudo foram similares às observadas por outros autores $^{(5,11)}$.

O efeito aprendizado na perimetria consiste na melhora da qualidade das informações fornecidas pelo paciente durante a confecção de exames seriados. A prática clínica nos mostra que indivíduos inexperientes na realização da perimetria podem apresentar alterações que não correspondem à realidade. A porcentagem de indivíduos normais que apresentaram efeito aprendizado utilizando o FDT (programa "screening test" C20-5) foi avaliada(21). Dos 81 indivíduos estudados, foram encontrados 12 (14,8\%) com exames alterados ou não confiáveis no primeiro teste realizado. Destes, 9 indivíduos $(11,1 \%)$ necessitaram de um segundo teste e 2 indivíduos (2,5\%) necessitaram ainda de um terceiro ou quarto teste para tornarem-se inalterados ou confiáveis, sendo que um indivíduo $(1,2 \%)$ ainda 
assim não apresentou exame normal no quarto teste. Em estudos que investigaram a perimetria acromática ${ }^{(2,22)}$ encontrou-se uma melhora significativa do MD, sugerindo a presença de um efeito aprendizado que poderia justificar uma etapa de treinamento antes da realização de perimetrias seqüenciadas. Iester et al. ${ }^{(5)}$ também analisaram o efeito aprendizado do FDT em quatro sessões diferentes e observaram que a média do MD em cada sessão foi de $-0,7 \pm 2,8 \mathrm{~dB}$, de $1,06 \pm 2,3 \mathrm{~dB}$, de $1,4 \pm$ $1,9 \mathrm{~dB}$ e de $1,2 \pm 1,3 \mathrm{~dB}$, respectivamente. Houve um aumento significativo do MD quando se comparou a primeira sessão com a segunda, terceira e quarta sessões. Nosso estudo confirma claramente um aumento da média do MD à medida que o paciente se torna mais experiente com esse tipo de perimetria. Entretanto, o aumento do MD não foi estatisticamente significante entre a primeira e a segunda sessões $(p=0,2127)$.

O presente estudo revelou que os limiares de sensibilidade detectados pela perimetria de freqüência dupla apresentaram flutuações a curto e longo prazos em indivíduos normais. Observou-se também um claro efeito aprendizado que deve ser considerado quando se realiza esse tipo de exame pela primeira vez. Essa curva de aprendizado justifica um treinamento inicial para que as informações fornecidas pelo paciente possam ser mais fidedignas.

A G R A D E C I M E N T O

À Prof. Tiemi Matsuo (Depto de Matemática Aplicada da Universidade Estadual de Londrina) pela realização dos cálculos estatísticos.

A B S T R A C T

Objective: To evaluate the learning effect, short-term fluctuation and long-term fluctuation in healthy subjects undergoing frequency doubling perimetry (FDP). Methods: Twenty healthy young subjects underwent FDT (program N30, full threshold) in one eye (right). Each subject was tested once in the first three sessions and three times in the fourth session. Both short- and long-term fluctuations were studied as the average fluctuation of all the tested points or as a point-to-point fluctuation. To study the learning effect, the MDs values of the first session were compared to the second, third and fourth sessions. Results: In the short-term analysis (3 examination done in the last session), the total mean sensitivity was $31.91 \pm 1.20 \mathrm{~dB}$ and the mean MD and PSD were $0.84 \pm 1.85$ and $3.73 \pm 1.55 \mathrm{~dB}$, respectively. The average short-term fluctuation was $1.72 \pm 0.38 \mathrm{~dB}$. When the four examination, performed at different visits, were compared, the average mean sensitivity of all sessions and the average longterm fluctuation were $31.75 \pm 1.11$ and $2.16 \pm 0.26 \mathrm{~dB}$, respectively. The MD averages of the first, second, third and fourth tests were $0.11 \pm 2.14 \mathrm{~dB}, 0.47 \pm 1.64 \mathrm{~dB}, 1.16 \pm 1.62 \mathrm{~dB}$ and $0.98 \pm 1.92 \mathrm{~dB}$ respectively. The MD difference between the first and the third and between the first and the fourth examinations were statistically significant $(\mathrm{p}<0.05)$. Conclusion: The threshold sensitivity detected by FDP is influenced by both short- and long-term fluctuations. We observed a mild learning effect that shoud be taken into account whenever a patient undergoes this test for the first time.

Keywords: Perimetry/methods; Visual fields/physiology; Learning

REFERÊNCIAS

1. Flammer J, Drance SM, Zulauf M. Differencial light threshold. Short and long-term fluctuation in patients with glaucoma, normal controls and patients with suspected glaucoma. Arch Ophthalmol 1984;102:704-6.

2. Wood JM, Wild JM, Hussey MK, Crews SJ. Serial examination of the normal visual field using Octopus automated projection perimetry. Evidence for a learning effect. Acta Ophthalmol 1987;65:326-33.

3. Heijl A, Bengtssom B. The effect of perimetric experience in patients with glaucoma. Arch Ophthalmol 1996;114:19-22.

4. Chauhan BC, Johnson CA. Test-retest variability of frequency-doubling perimetry and conventional perimetry in glaucoma patients and normal subjects. Invest Ophthalmol Vis Sci. 1999;40:648-56.

5. Iester M, Capris P, Pandolfo A, Zingirian M, Traverso CE. Learning effect, short-term fluctuation, and long-term fluctuation in frequency doubling technique. Am J Ophthalmol 2000;130:160-4.

6. Costa VP, Carvalho CA. Perimetria computadorizada. Um guia básico de interpretação. 2a. ed. Rio de Janeiro: RioMed; 2000.

7. Alward WL. Frequency doubling technology perimetry for the detection of glaucomatous visual field loss. Am J Ophthalmol 2000;129:376-8.

8. Johnson CA, Samuels SJ. Screening for glaucomatous visual field loss with frequency-doubling perimetry. Invest Ophthalmol Vis Sci. 1997;38:413-25.

9. Johnson CA, Wall M, Fingeret M, Lalle P. A Primer for Frequency Doubling Tecnology. Humphprey Manual.Rev. B 12/1998.

10. Quigley HA. Identification of glaucoma-related visual field abnormality with the screening protocol of frequency doubling technology. Am J Ophthalmol 1998; 125:819-29.

11. Cello KE, Nelson-Quigg JM, Johnson CA. Frequency doubling technology perimetry for detection of glaucomatous visual field loss. Am J Ophthalmol 2000;129:314-22.

12. Sponsel WE, Arango S, Trigo Y, Mensah J. Clinical classification of glaucomatous visual field loss by frequency doubling perimetry. Am J Ophthalmol 1998;125:830-6.

13. Burnstein Y, Ellish NJ, Magbalon M, Higginbotham EJ. Comparison of frequency doubling perimetry with Humphrey visual field analysis in a glaucoma practice. Am J Ophthalmol 2000;129:328-33.

14. Yamada N, Chen PP, Mills RP, Leen MM, Lieberman MF, Stamper RL, Stanford DC. Screening for glaucoma with frequency doubling technology and Damato campimetry. Arch Ophthalmol 1999;117:1479-84.

15. Patel SC, Friedmam DS, Varadkar P, Robin AL. Algorithm for interpreting the results of frequency doubling perimetry. Am J Ophthalmol 2000;129:323-7.

16. Bengtsson B, Lindgren A, Heijl A, Lindgren G, Asman P, Patella M. Perimetric probability maps to separate change caused by glaucoma from that caused by cataract. Acta Ophthalmol Scand 1997;75:184-8.

17. Haas A, Flammer J, Schneider U. Influence of age on the visual fields of normal subjects. Am J Ophthalmol 1986;101:199-203.

18. Katz J, Sommer A. A longitudinal study of the age - adjusted variability of automated visual fields. Arch Ophthalmol 1987;105:1083-6.

19. Iester M, Mermoud A, Schnyder C. Frequency doubling technique in patients with ocular hypertension and glaucoma: correlation with Octopus perimeter indices. Ophthalmology 2000;107:288-94.

20. Archibald SK, Hutchings N, Killoran JA, Flanagan JG. The magnitude of short and long-term fluctuation in young normal subjects for frequency doubling automated perimetry [abstract ]. Invest Ophthalmol Vis Science 2000; 41:S284.

21. Joson PJ, Kamantigue ME, Chen PP. Learning effects among perimetric novices in frequency doubling technology perimetry. Ophthalmology 2002; 109:757-60.

22. Marchini G, Pisano F, Bergtagnin F, Maraffa M, Bonomi L. Perimetric learning effects in glaucoma patients. Glaucoma 1991;13:102-6. 